\title{
Autophagy Inhibits Renal Tubule Inflammation in Diabetic Kidney Disease Mice Via Inhibition of NLRP3 Inflammasome
}

Pang Xinxin ( $\square$ doctorpang@aliyun.com )

Henan Provincial Hospital of Traditional Chinese Medicine https://orcid.org/0000-0001-7941-4860

\section{Ya-ge Zhang}

Henan Provincial Hospital of Traditional Chinese Medicine

\section{Li-jie Zhang}

First Affiliated Hospital of Zhengzhou University

Qing Zhu

Henan Provincial Hospital of Traditional Chinese Medicine

\section{Hai-tao Shang}

Henan Provincial of Traditional Chinese Medicine

\section{Zhen-tao Wang}

Henan Provincial of Traditional Chinese Medicine

\section{Research}

Keywords: diabetic kidney disease, autophagy, inflammation, NLRP3

Posted Date: February 2nd, 2022

DOI: https://doi.org/10.21203/rs.3.rs-1280850/v1

License: (9) (i) This work is licensed under a Creative Commons Attribution 4.0 International License. Read Full License 


\section{Abstract \\ Background}

Diabetic kidney disease (DKD) has become the leading cause of chronic kidney disease and numerous reports have validated that renal tubule inflammation participate in DKD progression. However, the molecular mechanism underlying renal tubular inflammatory damage in DKD remains to be completely elucidated. The aim of this study is to investigate the effect of rapamycin-induced autophagy on Nod-like receptor family protein 3 (NLRP3) inflammasome and inflammation of renal tubular epithelial cells in DKD.

\section{Methods}

We collected kidney tissue specimens from patients with DKD and transplanted kidney donors for histopathological examination and performed urine and blood samples. The STZ-induced DKD mice model was established for in vivo study. The mice were divided into three groups: Ctrl, DKD, DKD+Rapa. Collected the serum for $24 \mathrm{~h}$ protein quantification, blood glucose, C-peptide and insulin level investigations. PAS staining and electron microscopy were used to visualize the pathological changes in the kidney and the expressions of Beclin1, P62, NLRP3, apoptosis-associated speck-like protein containing a caspase recruitment domain (ASC) protein, interleukin (IL)-1 $\beta, I L-18, \beta 2$-microglobulin ( $\beta 2$ $M G) ; 24$ urine protein, and creatinine in the kidney tissue were detected. Moreover, human renal proximal tubule cells (HK-2) were cultured in vitro, and high glucose was used to induce the model, followed by rapamycin treatment. The protein expression of Beclin1, P62, ASC protein, IL-1ß, IL-18, 24 urine protein, and creatinine were analyzed.

\section{Results}

Compared with the model group mice, after rapamycin treatment, urinary protein decreased quantitatively, blood glucose decreased, C-peptide and insulin levels increased $(P<0.01)$, glomerular basement membrane did not thicken obviously, and the expressions of P62, NLRP3, ASC protein, IL-1 $\beta$ and IL-18 protein in renal tissue decreased, while Beclin-1 level increased $(P<0.05)$. After high glucose intervention in vitro, the expression of NLRP3, ASC protein, IL-1 $\beta$ and IL-18 protein in HK-2 cells increased, while the level of Beclin-1 decreased. The expression of NLRP3, ASC, IL-1 $\beta$ and IL-18 decreased and Beclin-1 level increased after rapamycin intervention $(P<0.05)$.

\section{Conclusion}

The findings of the present study indicated that the NLRP3 inflammasomemediated inflammatory response was activated in DKD. Rapamycin can inhibit the activation of NLRP3 inflammasome in DKD by 


\section{Introduction}

Diabetic kidney disease (DKD) is one of the most common microvascular complications of diabetes, affecting $30 \%-40 \%$ of people with type 2 diabetesand ithas become the leading cause of chronic kidney disease (CKD) with the increasing incidence of diabetes ${ }^{[1]}$. Classically, proteinuria was believed as an early marker of DKD onset or a sign of the severity of glomerular damage; however, growing evidence indicates that renal tubular damage is themajor causative factor in initiating and/or amplifying the onset of DKD complications ${ }^{[2]}$. In patients with diabetes, there is a predominance of increased levels of inflammatory mediators, and these mediators are thought to initiate the inflammatory cascade that leads to renal tubular damage, which further induces the generation of a large number of chemokines, adhesion factors, and pro-inflammatory cytokines, and aggravating the deterioration of renal function ${ }^{[3]}$. However,the molecular mechanism underlying renal tubular inflammatory damage in DKD remains to be completely elucidated.

Nod-like receptor family protein 3 (NLRP3) inflammasomes play different roles in innate immune signaling against pathogenic microbes and other endogenous or exogenous pathogens ${ }^{[4]}$. It has been found that the NLRP3 inflammasome is intimately involved in the inflammatory damage of renal tubular cells in DKD ${ }^{[5]}$. Upon stimulation by a plethora of stimuli, including high glucose, NLRP3 inflammasome recruits and activates procaspase- 1 to produce active caspase- 1 and then converts the cytokine precursorspro-interleukin (IL)-1 $\beta$ and pro-IL-18into mature and biologically active IL-1 $\beta$ and IL-18, respectively. Upon activation, mature IL-1 $\beta$ and IL-18 ${ }^{[6]}$ ead toa sequence of inflammatory responses involved in renal tubular injury in $D K D^{[7-9]}$.

Autophagy is a lysosome-dependent cellular degradation process that can maintain and regulate cell homeostasis by degrading intracellular components and providing cells with degradation products for the synthesis of macromolecules. Previous studies have confirmed that autophagy is dysregulated in DKD, implying that autophagy may be necessary for renal tubular homeostasis. Sun et al. ${ }^{[10]_{\text {found }} \text { that }}$ autophagy defects in distal renal tubular epithelial cells can lead to mitochondrial damage and increased oxidative stress, thereby activating the NLRP3 inflammasome/caspase-1/IL-1 $\beta$ signaling pathway and inducing apoptosis. This indicates that autophagy is associated with the activation of NLRP3 inflammasomes in damaged kidneys. However, it remains unclear whether autophagy can antagonize the inflammatory renal tubular injury in DKD by inhibiting the NLRP3 inflammasome. Therefore, is the present study aimed to investigate whether NLRP3 inflammasomes in DKD renal tubules were activated both in vivo and in vitro and the role of autophagy in regulating the inflammatory response in renal tubular epithelial cells activated by NLRP3 inflammasomes in DKD.

\section{Materials And Methods}




\section{Human Kidney Biopsy Samples And Urine Specimens}

Kidney biopsy samples and urine specimens were collected from 6 patients diagnosed with DKD at the Department of Nephrology, Henan Provincial Hospital of Traditional Chinese Medicine. Besides, samples from 6 non-DKD kidney transplantation donors were also collected as normal controls. All biopsy specimens were evaluated by a pathologist who was blinded to the results of molecular studies. The levels of IL-18 (abcam; ab215539), IL-1 $\beta$ (abcam; ab214025), and $\beta 2$-microglobulin ( $\beta 2-M G)$ (enzyme immunity; MM-0704H2) in urine samples were detected with an ELISA kit according to the manufacturers' instructions.

\section{Animal Studies}

All animals were cared for in accordance with the National Institute of Health (NIH) Guide for the Care and Use of Laboratory Animals. All procedures and animal experiments were approved by the Laboratory Animal Ethics Committee of the Henan Provincial Hospital of Traditional Chinese Medicine.

Forty SPF male C57BL/6N mice (23 $\pm 2 \mathrm{~g}$, certificate number: SCXK (Yu) 2017-0001) were procured from Henan Experimental Animal Center. The mice were housed with 3 animals per cage under specific pathogen-free conditions, at a temperature of $2423 \pm 2^{\circ} \mathrm{C}$ with a relative humidity of $40 \%-50 \%$, under a 12-h-light/12-h-dark schedule. Animals were provided ad libitum access to standard rodent food and tap water. All the mice were healthy and had no infection during the experimental period. Forty mice were fed adaptively for 1 week, the blood glucose was normal, and the urine protein qualitative test was negative. The mice were randomly divided into two groups; 10 mice in the control group $(n=10)$, and the remaining 30 mice were included in the experimental group. For the induction of diabetes, experimental group mice were fasted overnight and then administered with a single intraperitoneal injection of $60 \mathrm{mg} / \mathrm{kg} \mathrm{STZ}$ dissolved in $10 \mathrm{mM}$ citrate buffer $(\mathrm{pH}=4.5)$. The control group mice were only injected with an equal amount of citrate buffer. The blood glucose level of $\geq 16.7 \mathrm{mmol} / \mathrm{I}$ measured at $72 \mathrm{~h}$ indicated successful establishment of the animal model of diabetes. Of the 30 mice, 28 mice had blood glucose up to the standard. At week 4, a 24-hour urinary albumin excretion rate of $>30 \mathrm{mg} / 24 \mathrm{~h}$ indicated the successful construction of the DKD model ${ }^{[10]}$. Of the 28 mice, 24 reached the standard. The successful DKD animal models were randomly divided into the DKD experimental group $(n=12)$ and $D K D+R a p a$ group $(n=12)$. The DKD+Rapa group was administered with Rapamycin subcutaneous injection, and both the control group and the experimental group were administered normal saline $5 \mathrm{mg} / \mathrm{kg}$ by gavage/day. All animals were given continuous administration for 16 weeks, and then mice were sacrificed, and specimens were collected.

\section{Ethical statement}

This study was approved by the Ethics Committee of Henan Provincial Hospital of Traditional Chinese Medicine and was conducted in accordance with the Helsinki Declaration, and written informed consent was obtained from each participant. 


\section{Cell culture}

HK-2 cells were purchased from ATCC. After resuscitation, HK-2 cells were cultured in a DMEM/F12 medium supplemented with $10 \%$ fetal bovine serum (FBS; Gibco, Invitrogen) and 1\% penicillin/streptomycin at $37^{\circ} \mathrm{C}$ with $5 \% \mathrm{CO}_{2}$ in a humidified atmosphere.

At $80 \%-90 \%$ confluence, cells were digested with trypsin, and serum was added to the culture medium to terminate the digestion, and subsequently passed at a split ratio of $1: 3$, and plated for subsequent intervention. HK-2 cells were inoculated in a complete medium containing $10 \%$ fetal calf serum at $80 \%$ confluency for the high glucose treatment. After 24 hours, the culture medium was replaced with a serumfree medium and incubated for 16 hours. Subsequently, cells were treated with different concentrations of high sugar for a different time, and cells were then collected for further index detection.

\section{Serum biochemical measurements and blood sugar analysis}

Blood samples were collected from the tail vein every 4 weeks, and the blood glucose levels in the mice were measured with the Abbott glucometer (Abbott, USA) and its matching blood glucose test paper. The 24-hour urine samples from mice of each group were collected 1 day before sacrifice, and the 24-hour urine protein content was determined by the albumin determination kit (Nanjing Jiancheng Institute of Biological Engineering, Item No.: A028-1-1). The mice were weighed and fasted for $12 \mathrm{~h}$ before being sacrificed. Arterial blood samples were collected from the abdominal aorta under $10 \%$ chloral hydrate anesthesia. The serum insulinand C-peptide levels were assayed using commercially available kits.

\section{Pathological observation of kidney tissue}

Part of the kidney tissue specimens was fixed with $4 \%$ paraformaldehyde, embedded in paraffin, and sectioned into 5- $\mu \mathrm{m}$ sections. After drying, deparaffinization, dehydration in gradient alcohol, and rinsing with water, the sections were subjected to PAS staining. The pathological alterations in the kidney tissues from each group were observed under a light microscope. Moreover, part of the kidney tissue specimens was fixed with $2.5 \%$ glutaraldehyde, dehydrated, embedded, and sliced, and the renal tubules were observed under the transmission electron microscope.

Immunohistochemistry (IHC) Assay

For IHC, $4 \mu \mathrm{m}$ paraffin sections were deparaffinized in $100 \%$ xylene and rehydrated in an ethanol gradient. Antigen retrieval was performed by heating the tissue sections at $95-98^{\circ} \mathrm{C}$ in citrate buffer in a microwave oven for $15 \mathrm{~min}$. The endogenous peroxidase activity was blocked by $3 \% \mathrm{H}_{2} \mathrm{O}_{2}$ for 10 min and serum (5\% goat serum) for $30 \mathrm{~min}$ at $37^{\circ} \mathrm{C}$.Subsequently, the specimens were incubated with the appropriate amount of primary antibody IL-1 $\beta$ (1:100; CST, catalog number: \#12242S), IL-18 (1:200; abcam, article number: ab223293), caspase-1 (1:200; proteintech, article number: 22915-1-AP), Beclin-1 (1:250; proteintech, article number: 11306-1-AP), and P62 (1:150; proteintech, article number: 18420-1-AP), overnight at $4^{\circ} \mathrm{C}$. The sections were then washed thrice with PBS and incubated with secondary antibody and incubated at 
$37^{\circ} \mathrm{C}$ for $30 \mathrm{~min}$. The immunostaining was carried out by staining with $3,3^{\prime}$-diaminobenzidine chromogen (DAB)counter-stained with hematoxylin, dehydrated, and mounted, and the sections were examined under the microscope.

\section{Western blot assay}

The cultured and treated cells or tissue samples were collected after being washed with PBS. The total protein was extracted with RIPA lysis buffer (P0013K, Beyotime, China) supplemented with protease inhibitors cocktail on ice for 30 min. Protein concentration was quantified using the BCA method. An equal amount of protein was separated on $12 \%$ sodium dodecyl sulfate-polyacrylamide (SDS-PAGE), and then the proteins were electrophoretically transferred onto nitrocellulose (NC) membranes. Skimmed milk (5\%) was used to block heterogenetic antigen on the membranes for $2 \mathrm{~h}$. Subsequently, the membrane was incubated with primary antibodies against $\beta$-actin (1:4000; proteintech, article number: 60008-1-lg), NLRP3 (1: 1000; abcam, article number: ab48394), apoptosis-associated speck-like protein containing a caspase recruitment domain (ASC) (1:1000; CST, article number: \#67824), caspase-1 (1: 2000; proteintech, article number: 22915-1-AP), IL-1 $\beta$ (1:1000; CST, article number: \#12242S), IL-18 (proteintech, article number: 60070-1-lg), Beclin-1 (1: 2000; proteintech, product number: 11306-1-AP), and P62 (1:2000; proteintech, product number: 18420-1-AP), overnight at $4{ }^{\circ} \mathrm{C}$, After washing 5 times with TBST, 5 min each time, membranes were incubated with secondary antibodies (1: 2000), for $1 \mathrm{~h}$ at room temperature. The protein bands were visualized using the enhanced chemiluminescence detection kit, and the protein bands were imaged and analyzed using ImageJ software.

\section{Immunofluorescence}

The sections were fixed with4 \% paraformaldehyde for $30 \mathrm{~min}$ at room temperature. After fixation, the sections were washed with PBS and permeabilized with PBS containing $0.5 \%$ Triton X-100 for 30 min. Subsequently, sections were treated with $0.1 \mathrm{M}$ Tris- $\mathrm{HCl}(\mathrm{pH} 8.0)$ for $15 \mathrm{~min}$ and incubated in PBS containing $5 \%$ goat serum for 1 hour at $37^{\circ} \mathrm{C}$. Next, the sections were probed with different primary antibodies including IL-1 $\beta$ (1:100; proteintech, catalog number: 16806-1-AP), IL-18 (1:100; proteintech, article number: 10663-1-AP), and caspase-1 (1:100; proteintech, article number: 22915-1-AP) overnight at

$4{ }^{\circ} \mathrm{C}$. The sections were washed with PBS three times, followed by incubation with FITC-labeled secondary antibodies $(1: 100)$ for 1 hour in the dark at $37^{\circ} \mathrm{C}$. Then, sections were stained with DAPI to visualize the nucleusand observed under an Inverted Fluorescent Microscope.

\section{Statistical Analysis}

Data were expressed as mean \pm SD and were obtained from three independent experiments. The comparison between the two groups was performed using Student's t-test, and for comparison in multiple groups, one-way analysis of variancewas used. All statistical analyses were performed using SPSS 19.0 software (SPSS, Inc., Chicago, IL). A $P$-value of $<0.05$ was considered statistically significant.

\section{Result}


1. Urinary inflammatory factors in diabetic patients were significantly increased, NLRP3 inflammasomes in kidney tissue samples were activated, and autophagy was inhibited

To determine whether the inflammasome was activated in DKD and whether autophagy was attenuated, we examined the immunohistochemical staining intensity and distribution of NLRP3 and Beclin1 in the kidneys of patients with DKD and non-diabetic kidney disease. HE staining revealed that the renal tubules and glomeruli in patients with DKD exhibited apparent lesions, the glomerular volume was increased, the mesangial area was widened, the mesangial matrix increased, the renal tubular epithelial cells showed vacuolar degeneration and interstitial inflammatory cell infiltration. The PAS-positive staining in the kidney tissue of patients with DKD increased significantly: PASM staining and Masson staining showed mesangial hyperplasia and basement membrane thickening in patients with DKD; the structure of the kidney in patients with non-diabetic kidney disease under the electron microscope was normal with uniform glomerular basement membrane thickness. The protrusions were arranged in an orderly manner. In the DKD group, the foot protrusions were significantly widened and merged, and the glomerular basement membrane was unevenly thickened, as illustrated in Figure 1. An increased NLRP3 is an indication of inflammasome activation, and a decreased Beclin1 and P62 is an indication of autophagy inhibition. The results showed that in the kidney biopsy specimens of DKD, increased expression of NLRP3 inflammasome protein and IL-1 $\beta$ were observed, while the expression of autophagy marker Beclin1 was inhibited, and the expression of P62 was up-regulated, indicating that autophagy was inhibited, as shown in Figure 2. At the same time, we investigated the urine inflammatory factors and renal function damage index $\beta 2-M G$ in patients with non-diabetic kidney disease and DKD and found that patients with diabetic nephropathy had significantly higher urine IL-1 $\beta$, urine IL-18 and $\beta 2-\mathrm{MG}$, and $24 \mathrm{~h}$ urine protein levels than transplanted kidney donors, and significantly reduced GFR, as shown in Table 1.

Table 1 Inflammatory and renal function indicators in patients with diabetic kidney disease and transplant donor nephropathy

\begin{tabular}{|lll|}
\hline Group & Non-DKD Group & DKD Group \\
\hline Urine IL-1 $\beta(\mathrm{ng} / \mathrm{L})$ & $69.94 \pm 8.36$ & $153.56 \pm 36.73^{\# \#}$ \\
\hline Urine IL-18 $(\mathrm{ng} / \mathrm{L})$ & $76.54 \pm 9.26$ & $145.31 \pm 33.72^{\# \#}$ \\
\hline Urine $\beta 2-\mathrm{MG}(\mathrm{mg} / \mathrm{L})$ & $5.33 \pm 1.37$ & $23.33 \pm 9.62^{\# \#}$ \\
\hline 24h urine protein(mg/24h) & $87.83 \pm 33.16$ & $4924.83 \pm 1674.24^{\# \#}$ \\
\hline GFR(ml-min-1·(1.73 m2囚-1) & $94.38 \pm 8.94$ & $56.64 \pm 10.67^{\# \#}$ \\
\hline
\end{tabular}

Note: Non-DKD: transplant kidney donor; DKD: patients with diabetic kidney disease. Results were expressed as mean \pm SD. \#\#: $P<0.01$, \#: $P<0.05$ versus Ctrl. 
2. Autophagy activation reversed kidney pathology, renal function, and inflammation indicators in diabetic mice

Special staining of kidney tissue showed that the structure of glomeruli and renal tubules in the control group was clear and complete. In the experimental group, the glomerulus volume was increased, collagen deposition was evident, the mesangial matrix proliferated, the mesangial area was widened, capillary loops were increased, and tubular epithelial cells exhibited swelling, fusion, necrosis, and pathological changes; however, in DKD+Rapa group, all changes significantly improved as illustrated in Figure 2. Transmission electron microscopy revealed that the structure of the kidney in the control group was normal with a uniform thickness of the glomerular basement membrane, orderly arranged podocytes, and morphologically normal mitochondria in the renal tubules. While the foot processes of the DKD experimental group were significantly enlarged and fused, and the glomerular basement was uneven, and membranes were thickened; however, swelling of mitochondria in the renal tubules and spine breakage in the DKD+Rapa group was significantly reduced compared with the DKD experimental group as shown in Figure 3.

After the last administration, compared with the control group, C-peptide and insulin in the DKD experimental group were significantly reduced $(P<0.01)$; compared with the DKD experimental group, Cpeptide and insulin in the DKD+Rapa group were significantly increased $(P<0.05)$; compared with the control group, $24 \mathrm{~h}$ Pro, fasting blood glucose (FBG), and Bgroup, $24 \mathrm{~h}$ Pro, the DKD experimental group were significantly increased $(P<0.01$. As compared with the DKD experimental group, the Scr, and BUN in the DKD+Rapa group both Pro and FBG were significantly reduced at $24 \mathrm{~h}(P<0.05, P<0.01)$; compared with thecontrol group, the inflammatory indicators of IL-1 and IL-18 of the DKD experimental group were significantly increased $(P<0.01)$. After treatment with rapamycin, compared with the DKD experimental group $(P<0.05, P<0.01)$, the results was shown in Table 2 .

Table 2 Renal function and inflammation index levels in mice of each group

\begin{tabular}{|c|c|c|c|}
\hline Group & Ctrl Group & $\begin{array}{l}\text { DKD experimental } \\
\text { Group }\end{array}$ & $\begin{array}{l}\text { DKD+Rapa } \\
\text { Group }\end{array}$ \\
\hline C-peptide $(\mathrm{pg} / \mathrm{ml})$ & $468.39 \pm 40.34$ & $372.03 \pm 31.63^{\# \#}$ & $437.70 \pm 42.32^{*}$ \\
\hline Insulin( $\mu \mu$ suli) & $9.94 \pm 0.96$ & $6.52 \pm 1.47^{\# \#}$ & $7.89 \pm 0.74^{*}$ \\
\hline $\begin{array}{l}\text { 24h urine protein } \\
\text { quantification }(\mathrm{mg} / 24 \mathrm{~h})\end{array}$ & $9.23 \pm 1.84$ & $45.90 \pm 10.03^{\# \#}$ & $17.29 \pm 8.86^{\star \star}$ \\
\hline Fasting blood glucose(mmol/L) & $9.16 \pm 5.25$ & $21.27 \pm 2.84^{\# \#}$ & $15.38 \pm 3.66^{*}$ \\
\hline Urine $\beta 2-\mathrm{MG}(\mathrm{mg} / \mathrm{L})$ & $4.89 \pm 2.67$ & $38.34 \pm 8.02^{\# \#}$ & $24.53 \pm 6.08^{\star \star}$ \\
\hline Urine IL-1 $\beta(\mathrm{ng} / \mathrm{L})$ & $20.93 \pm 2.07$ & $115.54 \pm 10.77^{\# \#}$ & $58.35 \pm 7.15^{\star \star}$ \\
\hline Urine IL-18(ng/L) & $42.07 \pm 3.55$ & $138.49 \pm 8.49^{\# \#}$ & $88.19 \pm 6.14^{\star \star}$ \\
\hline
\end{tabular}


Note: Ctrl: control mice; DKD: diabetic kidney disease mice; DKD+Rapa: diabetic kidney disease +

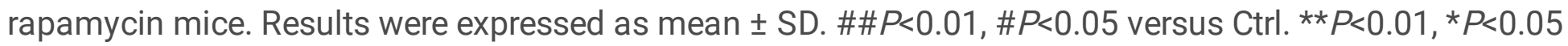
versus DKD.

3. Rapamycin inhibited the activation of inflammasomes in diabetic kidneys in mice and reduced the inflammatory response in kidney tissues

In order to confirm the effect of autophagy on the activation of kidney inflammasomes in DKD mice, we replicated the diabetic mouse model through STZ and used the autophagy activator, rapamycin to intervene, and found that the kidney IL-1 $\beta$, IL-18, the expression of caspase-1, and P62 were decreased, and the expression of Beclin-1 increased, suggesting that enhanced autophagy and inhibited inflammasome activation may be as an approach to reverse the pathology of DKD kidney (Figure 4 and Figure 5).

4. High glucose suppressed autophagy in HK-2 cells, triggered the activation of NLRP3 inflammasome in HK-2 cells, and promoted pyrolysis

As animal experiments have confirmed the correlation between autophagy and inflammasome activation, we speculated that autophagy inhibition plays a vital role in inflammasome activation. In order to verify this hypothesis, we first studied the expression of Beclin-1, P62, LC3 in HK-2 cells after treatment with high glucose. As presented in Figure 6 high glucose inhibited HK-2 cell autophagy in a dose-dependent manner. Since the NLRP3 inflammasome is currently the most characteristic inflammasome, we next investigated NLRP3 and ASC expression in HK-2 cells after treatment with high glucose. As depicted in Figure 7, Western blot analysis revealed that high glucose-induced NLRP3 and ASC expression in a doseand time-dependent manner.

5. Induction of autophagy by Rapamycin can reduce NLRP3 inflammasome activation

In order to further prove the significance of autophagy in regulating NLRP3 inflammasome activation, we used the known autophagy activator Rapamycin to induce autophagy in HK-2 cells. In the absence or presence of rapamycin, we treated HK-2 cells with high glucose. The results revealed that Rapamycin treatment could inhibit the activation of caspase- 1 induced by high glucose, as shown in Figure 8. Similarly, compared with the high sugar treatment group, the maturation of IL-1ßand IL-18 protein was also reduced after the pretreatment with rapamycin. Together, these data further confirmed that enhanced autophagy inhibited NLRP3 inflammasome activation under high glucose stimulation. The results of immunofluorescence were also consistent with this finding, as illustrated in Figure 9.

\section{Discussion}

The pathogenesis of DKD is highly complex, encompassing the accumulation of glycosylated end products, genetic factors, lipid metabolism, autophagy, and inflammation. The inflammatory responses are increasingly hypothesized to play a significant role in the renal tubular damage in DKD [11]. Studies 
have shown that in DKD, high glucose and other factors can activate the expression of renal tubular cells to secrete a variety of chemokines, adhesion molecules, and pro-inflammatory cytokines ${ }^{[12]}$. NLRP3 inflammasome, an essential member of the NOD-like receptor family, can recognize the danger signals generated by various risk factors in the human body, regulate the innate immune response, and recognize various pathogenic microbes (such as viruses and bacteria). Moreover, endogenous or exogenous (such as urate crystals) danger signals are the core components of the inflammatory response ${ }^{[4]}$. In this study, by detecting NLRP3 in the kidneys of DKD patients and kidney transplant donors, it was found that NLRP3 increased significantly, which indicated that NLRP3 corpuscles were activated in DKD patients.

In the environment of high sugar and other pathological factors, NLRP3 binds to the ASC protein, and the ASC protein recruits pro-caspase- 1 through its CARD domain, which cause NLRP3 inflammasome activation, and the pro-caspase- 1 is cut into Mature caspase-1, on the one hand,caspase-1 mediates the processing and maturation of IL-1 $\beta$ and IL-18 precursors, making them have pro-inflammatory functions, thereby causing tissue inflammatory damage, on the other hand, mediates the occurrence of pyrolysis characterized by plasma membrane rupture and the release of pro-inflammatory cell contents, and participates in the process of DKD disease ${ }^{[6,13]}$. More and more evidence emphasizes the importance of IL-1 $\beta$ and IL-18 in mediating renal tubular epithelial cell injury ${ }^{[7-9]}$. The results of this study also proved this view again. In DKD patients and in vivo and in vitro experiments, the expression of caspase-1 was upregulated, and the inflammation-related factors of IL-1 $\beta$ and IL-18 increased, indicating that the inflammatory system was significantly activated.

Different studies have also confirmed that autophagy can inhibit pyrolysis through negative regulation of NLRP3 inflammasomes ${ }^{[14]}$. In this study, in the HK-2 cell model induced by high glucose, it was found that after the enhancement of autophagy, the activation of caspase- 1 can be inhibited, and the expression of IL-1 $\beta$ and IL-18 can be down-regulated. The scorch caused by sugar stimulation further improves the damage of the kidney, which is also confirmed by the immunofluorescence results.

In recent years, studies have found that DKD is closely associated with autophagy dysfunction. Autophagy is a programmed degradation mechanism that maintains cellular metabolic balance, mainly by degrading the cytoplasm to achieve the cell's own metabolic needs and the renewal of certain organelles ${ }^{[15]}$.In DKD, under high glucose, hypoxia, toxic injury, oxidative stress, and other pathological conditions, the autophagy function of renal tubular cells is inhibited, which eventually leads to the accumulation of cell damage, leading to the development of $\mathrm{DKD}^{[16]}$. Zhuang et al. suggested that enhanced autophagy can prevent renal tubular epithelial cell damage caused by high glucose ${ }^{[17]}$. The expression of Becline- 1 is widely used to quantify the formation of autophagy, and P62 is the central marker of autophagy degradation ${ }^{[18]}$. in this study, it was found that the expression of Beclin 1 was inhibited and the expression of P62 was up-regulated in renal tubules in renal biopsy specimens of patients with DKD. At the same time, the same results were obtained in in vivo and in vitro experiments, which indicated that autophagy was obviously inhibited in DKD environment. 
Autophagy is closely related to inflammatory response. Autophagy inhibits the occurrence of inflammatory response through a variety of mechanisms. Studies have shown that autophagy-deficient kidney tissue accumulates damaged mitochondria, triggering mitophagy ${ }^{[19]}$. Renal tubules are rich in mitochondria. Mitochondria serve as energy metabolism organelles, and a large amount of reactive oxygen species produced by metabolism can trigger inflammatory responses ${ }^{[20]}$. In addition to mitochondria, autophagy can also prevent the rupture of lysosomes and thus inhibit the inflammatory damage caused by them ${ }^{[21]}$. As the core molecule of inflammatory response, NLRP3 is also related to autophagy. In the study of Xue et al. ${ }^{[22]}$, knockdown of LncRNA-Cox2 inhibits the activation of NLRP3 and can trigger the caspase-1/IL-1 $\beta$ cascade. To enhance autophagy, there is a crosstalk relationship between NLRP3 inflammasome and autophagy. Our study found that in DKD mice, by enhancing autophagy activity, it can significantly improve the pathological damage of glomeruli and renal tubules. Scr, BUN, $24 \mathrm{~h}$ Pro and FBG significantly decrease, indicating that the enhancement of autophagy has an effect on renal function and renal function. The morphology has been improved. In addition, after intervention with rapamycin, the expression of caspase-1, IL-1 $\beta$ and IL-18 was significantly downregulated, indicating that enhanced autophagycan significantly inhibit inflammation, and enhanced autophagy inhibits inflammasome activation It may be a way to improve the kidney pathology of DKD.

There are still some shortcomings in our research. The number of cases we have in humans is small, perhaps underrepresented; the autophagy system is relatively large, and some deeper mechanisms are still to be studied. In conclusion, we have proved that the renal tubular NLRP3 inflammasome is activated in DKD in vivo and in vitro. In addition, we also found that attenuation of autophagy plays an important role in the activation of inflammasomes. Therefore, autophagy is expected to become a new way to hinder the activation of NLRP3 inflammasomes and prevent the progression of DKD.

\section{Conclusion}

The findings of the study suggested that NLRP3 inflammasome-mediated inflammatory response is activated in DKD and the activation of NLRP3 inflammasome can be inhibited by rapamycinin DKD by enhancing autophagy.

\section{Abbreviations}




\begin{tabular}{|ll|}
\hline Abbreviations & Full names \\
\hline NLRP3 & Nod-like receptor family protein 3 \\
\hline DKD & diabetic kidney disease \\
\hline ASC & apoptosis-associated speck-like protein containing a caspase recruitment domain \\
\hline IL & interleukin \\
\hline HK-2 & B2-microglobulin \\
\hline CKD & human renal proximal tubule cells \\
\hline NIH & chronic kidney disease \\
\hline IHC & National Institute of Health \\
\hline DAB & Immunohistochemistry \\
\hline SDS-PAGE & diaminobenzidine chromogen \\
\hline NC & nodium dodecy I sulfate-polyacrylamide \\
\hline FBG & fasting blood glucose \\
\hline
\end{tabular}

\section{Declarations}

\section{Ethics approval and consent to participate}

All the animal experiments were approved by the ethics committee of Henan Hospital of Traditional Chinese Medicine

\section{Consent for publication}

Not Applicable. This article does not contain any studies with human participants performed by any of the authors.

\section{Availability of data and materials}

All data generated or analyzed during this study are included in this published article.

\section{Competing Interests}

The authors state that there are no conflicts of interest to disclose.

\section{Funding}


the Joint Construction Project of Henan Province (No. 2018020104); the Research Project of the National TCM Clinical Research Base (2019 JDZX068, 2019JDZX2119); the Key R \& D and Extension Project in HenanProvince(202102310505, 202102310171); A Sub-Station Project of the Inheritance Studio of Famous Old Chinese Medicine Experts Across the Country (none); the Special Research Project ofTCM in Henan Province (2019 ZYBJ17).

\section{Authors' Contributions}

P-XX designed and reviewed the manuscript. Z-YG and Z-IJ wrote the first draft of the paper. All authors read, revised and approved the final manuscript.

\section{Acknowledgements}

Our thanks go to all Laboratory members who helped to carry out this project.

\section{References}

[1] ZHANG L, LONG J, JIANG W, et al. Trends in Chronic Kidney Disease in China[J]. N Engl J Med, 2016,375(9): 905-906.

[2] ZENI L, NORDEN A, CANCARINI G, et al. A more tubulocentric view of diabetic kidney disease[J]. J Nephrol, 2017,30(6): 701-717.

[3] MATOBA K, TAKEDA Y, NAGAI Y, et al. Unraveling the Role of Inflammation in the Pathogenesis of Diabetic Kidney Disease[J]. Int J Mol Sci, 2019,20(14).

[4] MANGAN M, OLHAVA E J, ROUSH W R, et al. Targeting the NLRP3 inflammasome in inflammatory diseases[J]. Nat Rev DrugDiscov, 2018,17(8): 588-606.

[5] TANG S, YIU W H. Innate immunity in diabetic kidney disease[J]. Nat Rev Nephrol, 2020.

[6] JORGENSEN I, RAYAMAJHI M, MIAOEA. Programmed cell death as a defence against infection[J]. Nat Rev Immunol, 2017,17(3): 151-164.

[7] WETMORE J B, MAHNKEN J D, RIGLER S K, et al. Impact of race on cumulative exposure to antihypertensive medications in dialysis[J]. Am J Hypertens, 2013,26(2): 234-242.

[8] LIN J, CHENG A, CHENG K, et al. New Insights into the Mechanisms of Pyroptosis and Implications for Diabetic KidneyDisease[J]. Int J Mol Sci, 2020,21(19):7057.

[9] LIU P, ZHANG Z, LI Y. Relevance of the Pyroptosis-Related Inflammasome Pathway in the Pathogenesis ofDiabetic Kidney Disease[J]. Front Immunol, 2021,12: 603416.

[10] NAM S A, KIM W Y, KIM J W, et al. Autophagy attenuates tubulointerstital fibrosis through regulating transforminggrowth factor- $\beta$ and NLRP3 inflammasome signaling pathway[J]. Cell Death Dis, 2019,10(2): 
78.

[11] LI S, JIA Y, XUE M, et al. Inhibiting Rab27a in renal tubular epithelial cells attenuates the inflammation ofdiabetic kidney disease through the miR-26a-5p/CHAC1/NF-kB pathway[J]. Life Sci, 2020,261: 118347.

[12] ZOJA C, MORIGI M, REMUZZI G. Proteinuria and phenotypic change of proximal tubular cells[J]. J Am Soc Nephrol, 2003,14 Suppl 1: S36-S41.

[13] QIU Y Y, TANG L Q. Roles of the NLRP3 inflammasome in the pathogenesis of diabetic nephropathy[J]. Pharmacol Res, 2016,114: 251-264.

[14] HARRIS J, LANG T, THOMAS J, et al. Autophagy and inflammasomes[J]. Mol Immunol, 2017,86: 1015.

[15] PARZYCH K R, KLIONSKY D J. An overview of autophagy: morphology, mechanism, and regulation[J]. Antioxid Redox Signal, 2014,20(3): 460-473.

[16] DING Y, CHOI M E. Autophagy in diabetic nephropathy[J]. J Endocrinol, 2015,224(1): R15-R30.

[17] ZHUANG L, JIN G, HU X, et al. The inhibition of SGK1 suppresses epithelial-mesenchymal transition and promotesrenal tubular epithelial cell autophagy in diabetic nephropathy[J]. Am J Transl Res, 2019,11(8): 4946-4956.

[18] WU Z Z, ZHANG J J, GAO C C, et al. Expression of autophagy related genes mTOR, Becline-1, LC3 and p62 in the peripheralblood mononuclear cells of systemic lupus erythematosus[J]. Am J Clin Exp Immunol, 2017,6(1): 1-8.

[19] KIMURA T, TAKABATAKE Y, TAKAHASHI A, et al. Autophagy protects the proximal tubule from degeneration and acute ischemic injury[J]. J Am Soc Nephrol, 2011,22(5): 902-913.

[20] ZHOU R, YAZDI A S, MENU P, et al. A role for mitochondria in NLRP3 inflammasome activation[J]. Nature, 2011,469(7329): 221-225.

[21] KIMURA T, ISAKA Y, YOSHIMORI T. Autophagy and kidney inflammation[J]. Autophagy, 2017,13(6): 997-1003.

[22] XUE Z, ZHANG Z, LIU H, et al. lincRNA-Cox2 regulates NLRP3 inflammasome and autophagy mediated neuroinflammation[J]. Cell Death Differ, 2019,26(1): 130-145.

\section{Figures}

\section{Figure 1}


Kidney pathological changes in patients with diabetic kidney disease and non-diabetic kidney disease

Non-DKD: transplant kidney donor; DKD: diabetic kidney disease patients. Figure A shows the special staining of HE, PAS, MASSON, and PASM in patients with diabetic kidney disease and non-diabetic kidney disease (magnification $\times 200$ ). Figure $B$ shows the results of kidney electron microscopy in patients with diabetic kidney disease and non-diabetic kidney disease (magnification at 20000x).

$\begin{array}{llll}\text { Becline-1 } & \text { P62 } & \text { IL-1 } \beta & \text { NLRP3 }\end{array}$

\section{Figure 2}

Expression of tubular inflammation markers and autophagy in patients with diabetic kidney disease and transplant kidney donors (magnification at 400x)

Non-DKD: transplant kidney donor; DKD: patients with diabetic kidney disease.

Figure 3 


\section{Histological changes in mouse kidneys of each group}

Figure A PAS staining showed that the basement membrane of the tubules in the model group was thickened, and the proximal tubules were dilated. (magnification at 400X). Figure B The electron microscope results showed that the mitochondria of the renal tubules in the experimental group were swollen, and the spines were broken and disappeared. Ctrl: control group; DKD: diabetic kidney disease; Rapa: rapamycin.

A

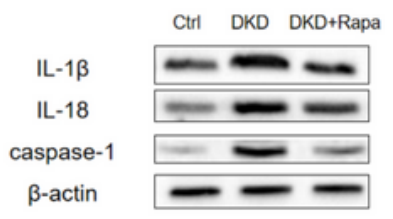

C

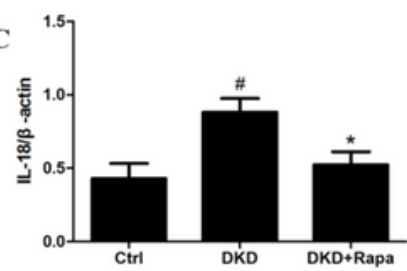

$\mathbf{E}$

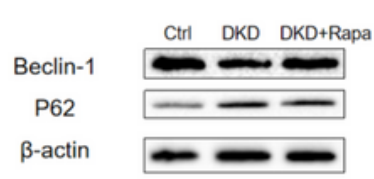

G

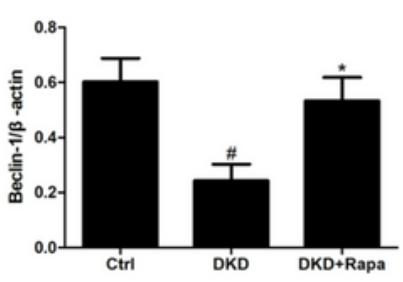

B

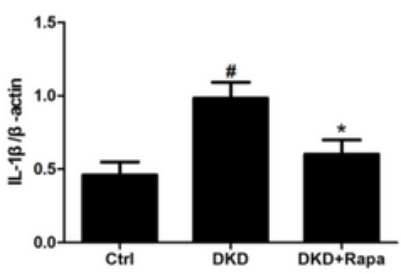

D

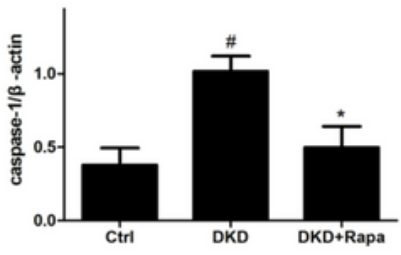

F

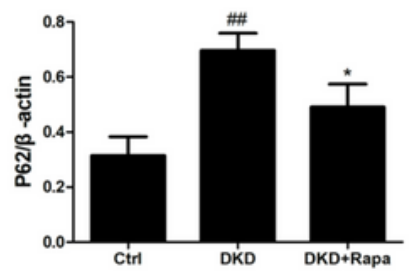


Figure 4

The effect of Rapamycin on the expression of autophagy markers and inflammasome protein in diabetic kidney disease mice

Figures A-D are Western blots analysis of IL-1 $1 \beta, \mathrm{IL}-18$, and caspase- 1 and related expression levels; E-F are Western blots analysis of Beclin1 and P62 and related expression levels. Data were expressed as mean \pm standard deviation. Ctrl: control mice; DKD: diabetic kidney disease mice; DKD+Rapa: diabetic kidney disease + rapamycin mice. Compared with the normal control group, \#\#: $P<0.01, \#$ : $P<0.05$; compared with the experimental group, $* *: P<0.01, *: P<0.05$. 


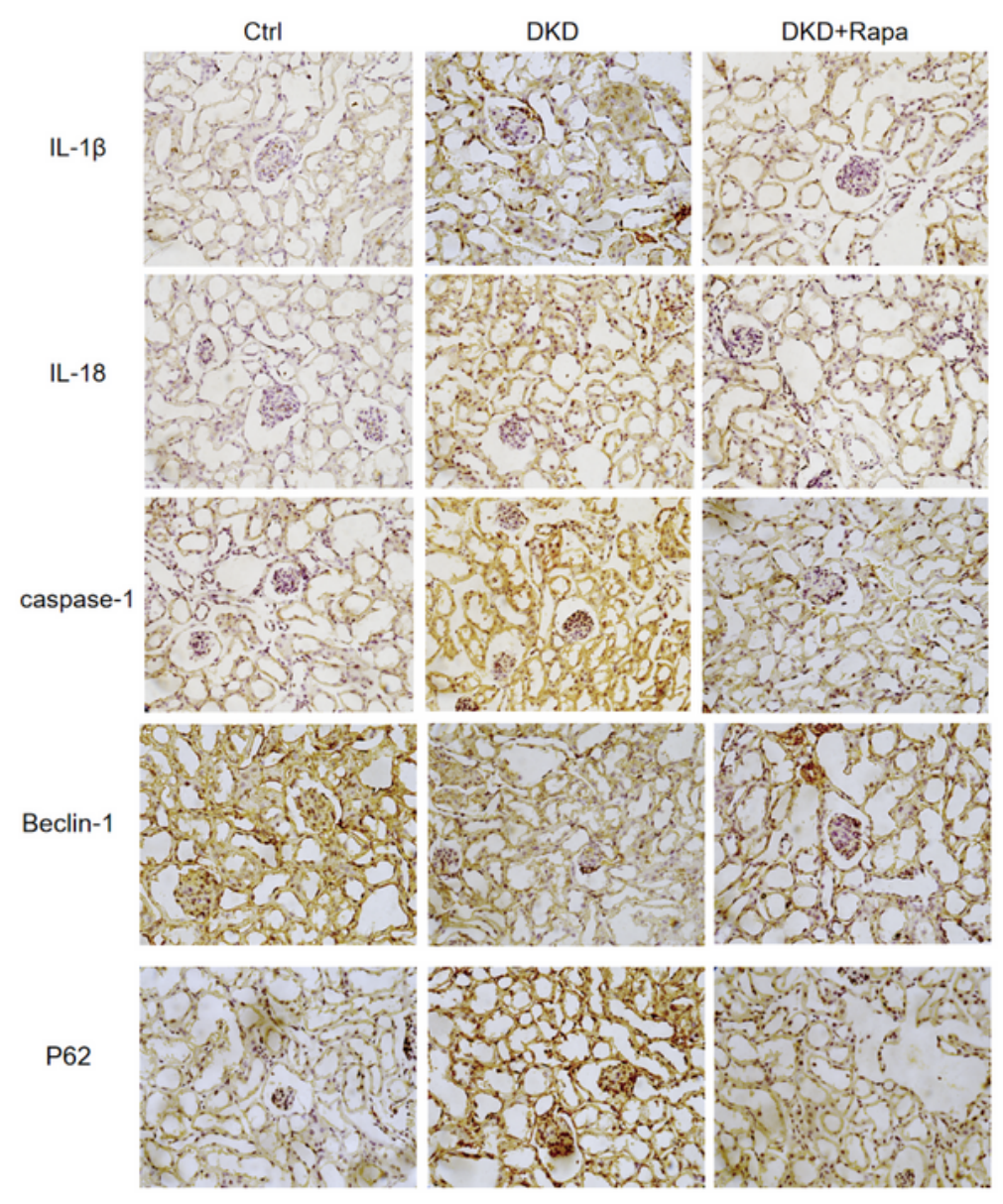

\section{Figure 5}

The effect of Rapamycin on the expression of autophagy markers and inflammasomes in diabetic kidney disease mice

Therepresentative images of the immunohistochemical analysis of autophagy markers and inflammasome-related proteins (magnification at 400X). Ctrl: control mice; DKD: diabetic kidney disease 
mice; DKD+Rapa: diabetic kidney disease + rapamycin mice.

Figure 6

Effects of different concentrations of high glucose on autophagy markers P62 andBeclin-1 in HK2 cells

Figures A-C Western blots analysis of Beclin1 and P62 and related expression levels. Data were expressed as mean and related expression levels. gh glucose on autop $30 \mathrm{mmol} / \mathrm{L}$ high glucose; HG2: $45 \mathrm{mmol} / \mathrm{L}$ high glucose; HG3: $60 \mathrm{mmol} / \mathrm{L}$ high glucose. Compared with the normal control group, \#\#: $P<0.01$, \#: $P<0.05$; compared with the experimental group, **: $P<0.01, *$ : $P<0.05$.
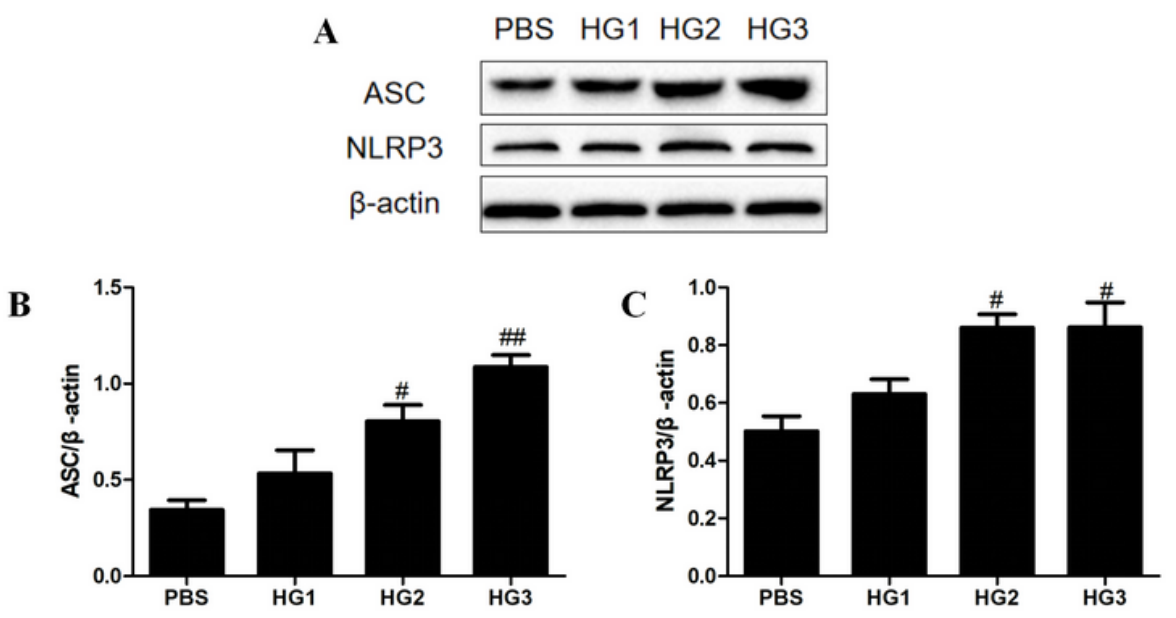

Figure 7

The effect of different concentrations of high glucose on HK2 cell pyrolysis markers

Figures A-C Western blot analysis of ASC and NLRP3 and their relative expression levels. Data were expressed as mean nd their relative expression levels. K2 cell pyrolysi mmol/L high glucose; HG2: 45 $\mathrm{mmol} / \mathrm{L}$ high glucose; HG3: $60 \mathrm{mmol} / \mathrm{L}$ high glucose. Compared with the normal control group, \#\#: $P<0.01$, \#: $P<0.05$; compared with the experimental group, $\star *: P<0.01, *: P<0.05$. 
Figure 8

The effect of Rapamycin on HK2 cells after treatment with high glucose

Figures A-D Western blot analysis of caspase1, IL-18, IL-1v and related expression levels. Data were expressed as mean ion levels. on HKion. PBS: control group HK2 cells; HG: $60 \mathrm{mmol} / \mathrm{L}$ high glucose; $\mathrm{HG}+$ Rapa: $60 \mathrm{mmol} / \mathrm{L}$ high glucose + rapamycin group. Compared with the normal control group, $\# \#: P<0.01, \#: P<0.05$; compared with the experimental group, $* *: P<0.01, *: P<0.05$.

A

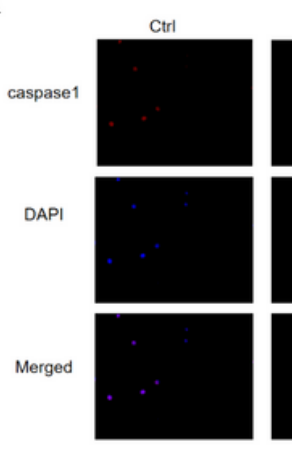

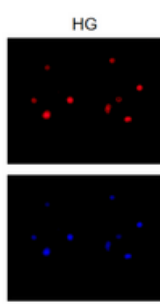

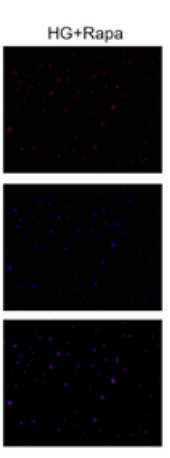

B
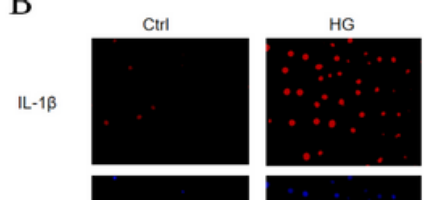

HG+Rapa

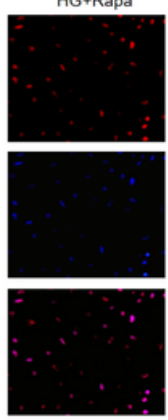

\section{Figure 9}

\section{The effect of Rapamycin on HK2 cells after treatment with high glucose}

Figures A-B immunofluorescence images of caspase 1 and IL-1 h tmagnification at 400x). PBS: control group HK2 cells; HG: $60 \mathrm{mmol} / \mathrm{L}$ high glucose; HG+Rapa: $60 \mathrm{mmol} / \mathrm{L}$ high glucose + rapamycin group. Compared with the normal control group, \#\#: $P<0.01$, \#: $P<0.05$; compared with the experimental group, $\star *: P<0.01, *: P<0.05$. 